\title{
Gravitational Wave (GW) Classification, Space GW Detection Sensitivities and AMIGO (Astrodynamical Middle-frequency Interferometric GW Observatory)
}

\author{
Wei-Tou $\mathrm{Ni}^{1}$ \\ Center for Gravitation and Cosmology (CGC), Department of Physics, National Tsing Hua \\ University, No. 101, Kuang Fu II Rd., Hsinchu, Taiwan, 300 ROC
}

\begin{abstract}
After first reviewing the gravitational wave (GW) spectral classification. we discuss the sensitivities of GW detection in space aimed at low frequency band $(100 \mathrm{nHz}-100 \mathrm{mHz})$ and middle frequency band $(100 \mathrm{mHz}-10 \mathrm{~Hz})$. The science goals are to detect GWs from (i) Supermassive Black Holes; (ii) Extreme-Mass-Ratio Black Hole Inspirals; (iii) Intermediate-Mass Black Holes; (iv) Galactic Compact Binaries; (v) Stellar-Size Black Hole Binaries; and (vi) Relic GW Background. The detector proposals have arm length ranging from 100 $\mathrm{km}$ to $1.35 \times 10^{9} \mathrm{~km}$ (9 AU) including (a) Solar orbiting detectors and (b) Earth orbiting detectors. We discuss especially the sensitivities in the frequency band $0.1-10 \mu \mathrm{Hz}$ and the middle frequency band $(0.1 \mathrm{~Hz}-10$ $\mathrm{Hz}$ ). We propose and discuss AMIGO as an Astrodynamical Middlefrequency Interferometric GW Observatory.
\end{abstract}

\section{Classification and Spectral Sensitivities}

With LIGO's direct detection of the binary black hole merger events $[1,2,3]$, we have been fully ushered into the age of Gravitational Wave (GW) astronomy. Detection efforts over all GW frequency bands from cosmological frequency band $(1 \mathrm{aHz}-10 \mathrm{fHz})$ to ultra-high frequency band (over $1 \mathrm{THz}$ ) have been vigorously exerted (See, e.g. [4]). In Table 1, we list the band ranges and the detection methods [4-6]. We have also plotted the GW detector sensitivities and GW source strengths on single diagrams with ordinates showing characteristic strain, strain power spectral density (psd) amplitude and normalized GW spectral energy density respectively in 2015 [4]. Currently we are updating these diagrams [7]. Fig. 1 shows the strain psd amplitude vs. frequency for various detectors and sources adapting from the corresponding figures of Refs. [4] and [7]. For detailed explanation of the plot, see [4, 7].

Presently, most GW detection efforts are spent in 4 bands - the high frequency band $(10 \mathrm{~Hz}-100 \mathrm{kHz})$, the upper part $(10 \mu \mathrm{Hz}-0.1 \mathrm{~Hz})$ of the low frequency band, the very low frequency band (nano- $\mathrm{Hz}$ band, $300 \mathrm{pHz}-100 \mathrm{nHz}$ ) and the extremely low (Hubble)

\footnotetext{
${ }^{1}$ weitou@gmail.com
} 
frequency band (cosmological band or CMB band; $1 \mathrm{aHz}-10 \mathrm{fHz}$ ). As can be seen in $\mathrm{Fi}_{\text {; }}$ 1 , there are 3 regions which are poor in the near-future projected sensitivities adjacent $t$ these 4 bands: (i) the middle frequency band, (ii) the lower part $(100 \mathrm{nHz}-10 \mu \mathrm{Hz})$ of th low frequency band and (iii) the ultralow frequency band $(10 \mathrm{fHz}-300 \mathrm{pHz})$. To possibl increase the sensitivity in the frequency band $0.1-10 \mu \mathrm{Hz}$, Super-ASTROD with arr length of $9 \mathrm{AU}$ has been proposed [8]. To have significant sensitivity in the frequenc: band $0.1-10 \mathrm{~Hz}$ and yet to be a first-generation candidate for space GW missions, w propose a middle-frequency GW mission AMIGO (Astrodynamical Middle-frequenc. Interferometric GW Observatory) with arm length $10,000 \mathrm{~km}$ and discuss the concept $\mathrm{i}$ : section 3 after a review on space GW detection sensitivities in section 2.

Table 1. Frequency classification of gravitational waves and their detection method [4-6]

\begin{tabular}{|l|l}
\hline Frequency band & Detection method \\
\hline \hline Ultra high frequency band: above $1 \mathrm{THz}$ & $\begin{array}{l}\text { Terahertz resonators, optical resonators, an } \\
\text { magnetic conversion detectors }\end{array}$ \\
\hline Very high frequency band: $100 \mathrm{kHz}-1 \mathrm{THz}$ & $\begin{array}{l}\text { Microwave resonator/wave guide detectors, lase } \\
\text { interferometers and Gaussian beam detectors }\end{array}$ \\
\hline $\begin{array}{c}\text { High frequency band (audio band)*: } 10 \mathrm{~Hz}- \\
100 \mathrm{kHz}\end{array}$ & $\begin{array}{l}\text { Low-temperature resonators and ground-base } \\
\text { laser-interferometric detectors }\end{array}$ \\
\hline Middle frequency band: $0.1 \mathrm{~Hz}-10 \mathrm{~Hz}$ & $\begin{array}{l}\text { Space laser-interferometric detectors of arr } \\
\text { length } 100 \mathrm{~km}-60,000 \mathrm{~km} \text {, atom and molecul } \\
\text { interferometry, optical clock detectors }\end{array}$ \\
\hline $\begin{array}{c}\text { Low frequency band (milli-Hz band) }{ }^{\dagger}: 100 \\
\text { nHz-0.1 Hz }\end{array}$ & $\begin{array}{l}\text { Radio Doppler tracking of spacecraft, spac } \\
\text { laser-interferometric detectors of arm lengt } \\
\text { longer than } 60,000 \mathrm{~km} \text {, optical clock detectors }\end{array}$ \\
\hline $\begin{array}{l}\text { Very low frequency band (nano-Hz band): } \\
300 \mathrm{pHz}-100 \mathrm{nHz}\end{array}$ & Pulsar timing arrays (PTAs) \\
\hline Ultralow frequency band: $10 \mathrm{fHz}-300 \mathrm{pHz}$ & Astrometry of quasars and their proper motions \\
\hline $\begin{array}{l}\text { Extremely low (Hubble) frequency band } \\
\text { (cosmological band): } 1 \mathrm{aHz}-10 \mathrm{fHz}\end{array}$ & Cosmic microwave background experiments \\
\hline $\begin{array}{l}\text { Beyond Hubble-frequency band: below 1 } 1 \\
\text { aHz }\end{array}$ & $\begin{array}{l}\text { Through the verifications } \\
\text { inflationary/primordial cosmological models }\end{array}$ \\
\hline
\end{tabular}

*The range of audio band (also called LIGO band) normally goes only to $10 \mathrm{kHz}$.

†The range of milli-Hz band is $0.1 \mathrm{mHz}$ to $100 \mathrm{mHz}$.

\section{SPACE GW detection sensitivities}

GW detection in space aimed at low frequency band $(100 \mathrm{nHz}-100 \mathrm{mHz})$ and middl frequency band $(100 \mathrm{mHz}-10 \mathrm{~Hz})$. Its scientific goals are to detect GWs from (i Supermassive Black Hole Coalescences; (ii) Extreme-Mass-Ratio Black Hole Inspirals (iii) Intermediate-Mass Black Hole Coalescences; (iv) Galactic Compact Binaries, (v Stellar-size Black Hole Binary Inspirals, and (vi) Relic GW Background.

The main technological requirements of GW detection in space are (i) drag-fre requirement; and (ii) requirement of measuring relative distance variation or relativ velocity variation. LISA Pathfinder (LPF) launched on 3 December 2015, has achiever not only the drag-free requirement goal of this technology demonstration mission, bu also has completely met the more stringent LISA drag-free demand [9-11]. In shor LISA Pathfinder has successfully demonstrated the first generation drag-free technolog. requirement for space detection of GWs. 


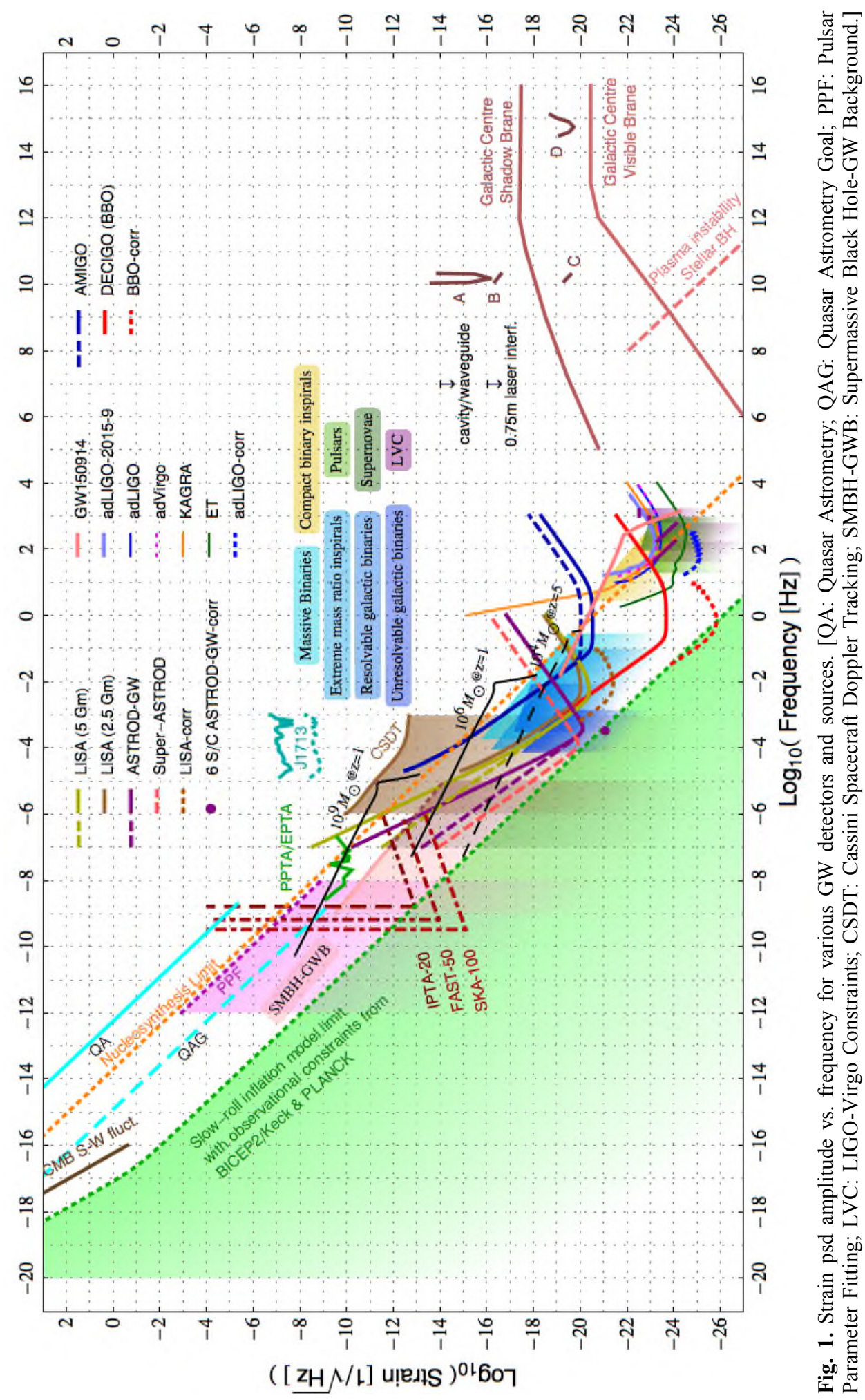


The requirement of measuring relative distance variation or relative velocity variation is in terms of spectral strain sensitivity. For space GW detection, the first-generation requirement is around $10^{-20} \mathrm{~Hz}^{-1 / 2}$ sensitivity for measurement of strain psd amplitude. For measurement using unequal-arm laser interferometry, the requirement on laser stabilization is similar. However, the present laser stabilization has not reached this kind of stability. One needs to match the two optical paths using Time Delay Interferometry (TDI) to lessen the stability requirement. For TDI configurations and their numerical simulations for various missions, see Tinto and Dhurandhar [12], Wang and Ni [13] and references therein. Experimental demonstration of TDI in laboratory for LISA worked out in 2010-2012 (Vine et al. [14], Mirtyk et al. [15]).

In space, Michelson type interferometry invariably involve large distances. The laser power received at the far end of the optical link is weak. To continue the optical path as required by TDIs, one needs to amplify it. The way of amplification is to track the optical phase of the incoming weak light with the local laser oscillator by optical phase-locking. At National Tsing Hua University, $2 \mathrm{pW}$ weak-light homodyne phase-locking with $0.2 \mathrm{~mW}$ local oscillator has been demonstrated (Liao et al. [16, 17]). In JPL (Jet Propulsion Laboratory), Dick et al. [18] have achieved offset phase locking of local oscillator to $40 \mathrm{fW}$ incoming laser light. More recently, Gerberding et al. [19] and Francis et al. [20] have phase-locked and tracked a $3.5 \mathrm{pW}$ weak light signal and a $30 \mathrm{fW}$ weak light signal respectively at reduced cycle slipping rate. For LISA, $85 \mathrm{pW}$ weak-light phase locking is required. For ASTROD-GW, $100 \mathrm{fW}$ weak-light phase locking is required. Hence, the weak level of these weak-light power requirements has achieved. In the future, the frequency-tracking, modulation-demodulation and coding-decoding needs development to make it a mature technology. This is also important for deep space CW (Continuous Wave) optical communication.

As shown in Fig. 1, typical frequency sensitivity spectrum of strain psd amplitude for space GW detection consists of three regions, the acceleration/local gravity gradient/vibration noise dominated region, the shot noise (flat for current space detector projects like LISA in strain psd) dominated region, if any, and the antenna response restricted region. The detector sensitivity in the lower frequency region is constrained by vibration, acceleration noise or gravity-gradient noise. The detector sensitivity of the higher frequency part is constrained by antenna response (or storage time). In a power-limited design, sometimes there is a middle flat region in which the sensitivity is limited by the photon shot noise. [21-24]

The shot noise sensitivity in the strain for GW detection is inversely proportional to $P^{1 / 2} L$ with $P$ the received power and $L$ the distance or arm length. Since $P$ is inversely proportional to $L^{2}$ and $P^{1 / 2} L$ is constant, this sensitivity limit is independent of the distance. For 1-2 W emitting power, the limit is around $10^{-20}-10^{-21} \mathrm{~Hz}^{-1 / 2}$ (depending on telescope diameter/laser beam divergence). As noted in the LISA study [21], making the arms longer shifts the time-integrated sensitivity curve to lower frequencies while leaving the bottom of the curve at the same level. Hence, ASTROD-GW with longer arm length has better sensitivity at lower frequency. e-LISA, ALIA, TAIJI, and GW interferometers in Earth orbit have shorter arms and therefore have better sensitivities at higher frequency.

In Fig. 1, we plot the sensitivity curves for LISA $(5 \mathrm{Gm})$, LISA $(2.5 \mathrm{Gm}$, i.e. the new LISA), ASTROD-GW, BBO, DECIGO, AMIGO and Super-ASTROD. The sensitivity curves for LISA (5 Gm), ASTROD-GW, BBO and DECIGO are taken from Fig. 3 of [4] and references therein. Others are from [7] and references therein. Fig. 2 is a blowup of Fig. 1 restricted to the frequency range $10^{-10}-10^{4} \mathrm{~Hz}$ to show the space frequency regions and the 2 neighboring regions. Section 2.1 discusses new LISA $(2.5 \mathrm{Gm})$ and gives its sensitivity equation. Section 2.2 does it for Super-ASTROD. 


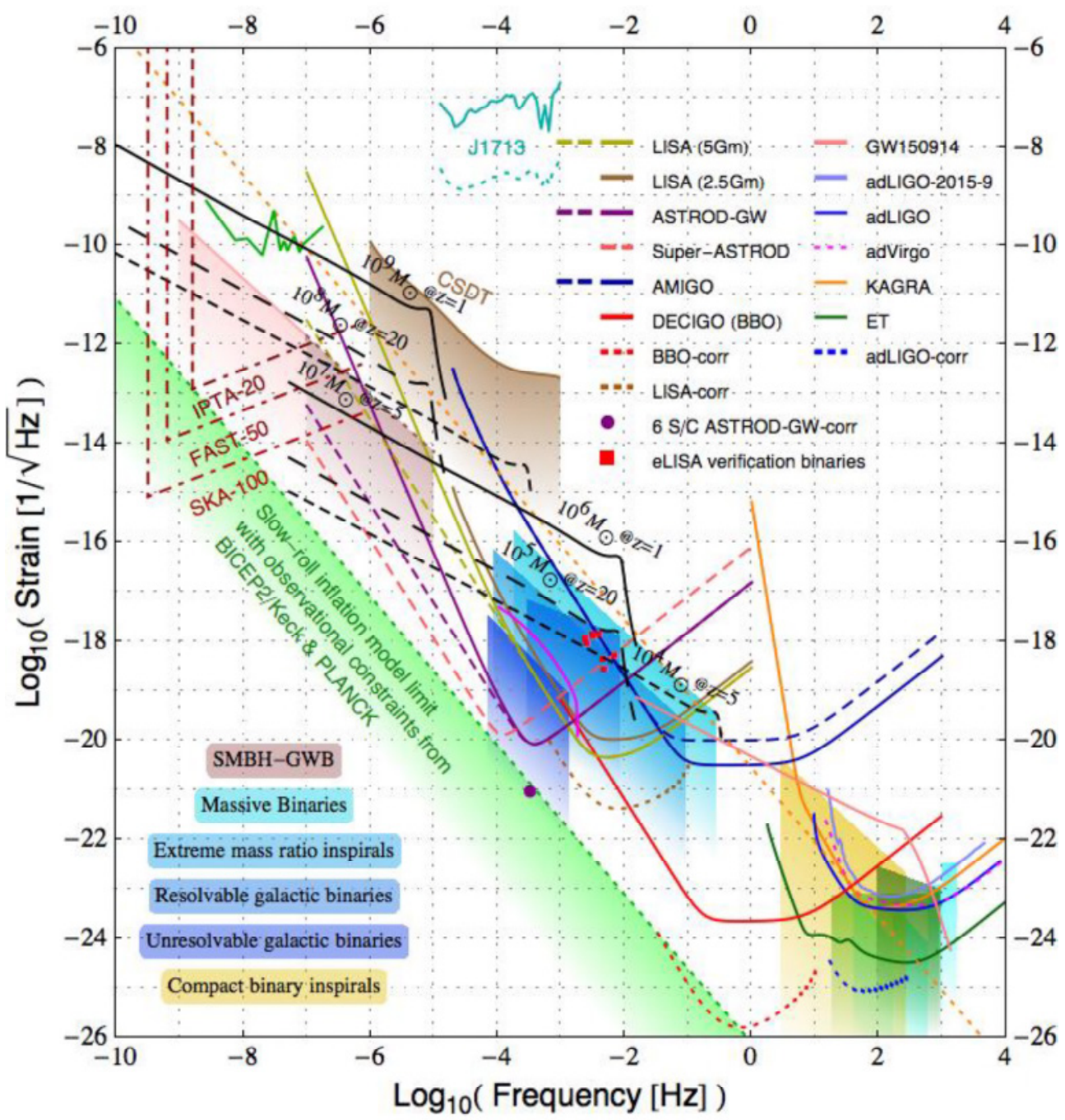

Fig. 2. Strain power spectral density (psd) amplitude vs. frequency for various GW detectors and GW sources. The black lines show the inspiral, coalescence and oscillation phases of GW emission from various equal-mass black-hole binary mergers in circular orbits at various redshift: solid line, $\mathrm{z}=1$; dashed line, $z=5$; long-dashed line $z=20$. See text of [24] for more explanation. The strain psd amplitude of GW150914 is calculated from its characteristic amplitude in Figure 1 of [25] using standard formula. The AMIGO design sensitivity is in solid blue while AMIGO baseline sensitivity is in dashed blue. The two curves merge together at lower frequency in the figure. [CSDT: Cassini Spacecraft Doppler Tracking; SMBH-GWB: Supermassive Black Hole-GW Background.]

\subsection{LISA (2.5 Gm)}

A new LISA proposal (Amaro-Seoane et al. [11]) was submitted to ESA on January 13th in response to the call for missions for the L3 slot in the Cosmic Vision Programme. On 20 June 2017, ESA announced the news that "The LISA trio of satellites to detect gravitational waves from space has been selected as the third large-class mission in ESA's Science programme (ESA 2017)." The basic concept is the same as the original LISA, but with arm length down-scaled to $2.5 \mathrm{Gm}$ from $5 \mathrm{Gm}$. To distinguish this selected mission proposal 
from the original one or the NGO/eLISA, we call it LISA $(2.5 \mathrm{Gm}$ or new LISA in case of possible ambiguity. Quoting from the proposal [11]:

"The observatory will be based on three arms with six active laser links, between three identical spacecraft in a triangular formation separated by 2.5 million $\mathrm{km}$. Continuously operating heterodyne laser interferometers measure with $\mathrm{pm} \mathrm{Hz}^{-1 / 2}$ sensitivity in both directions along each arm, using well-stabilized lasers at $1064 \mathrm{~nm}$ delivering $2 \mathrm{~W}$ of power to the optical system. Using technology proven in LISA Pathfinder, the Interferometry Measurement System is using optical benches in each spacecraft constructed from an ultralow expansion glass-ceramic to minimize optical path length changes due to temperature fluctuations. $30 \mathrm{~cm}$ telescopes transmit and receive the laser light to and from the other spacecraft. Three independent interferometric combinations of the light travel time between the test masses are possible, allowing, in data processing on the ground, the synthesis of two virtual Michelson interferometers plus a third null-stream, or "Sagnac" configuration." These two virtual Michelson interferometers are two TDIs. They could be two out of three TDI configurations $\mathrm{X}, \mathrm{Y}$ and $\mathrm{Z}$ if they satisfy the noise requirement.

The new LISA design sensitivity is in $[10,11]$. A simple analytical approximation of the design sensitivity is in Petiteau et al. [10] and used by Cornish and Robson [26]:

$$
S_{\mathrm{Ln}}^{1 / 2}(f)=(20 / 3)^{1 / 2}\left(1 / L_{\mathrm{L}}\right) \times\left[\left(1+\left(f /\left(1.29 f_{\mathrm{L}}\right)\right)^{2}\right)\right]^{1 / 2} \times\left[\left(S_{\mathrm{Lp}}+4 S_{\mathrm{a}} /(2 \pi f)^{4}\right)\right]^{1 / 2} \mathrm{~Hz}^{-1 / 2},
$$

over the frequency range $20 \mu \mathrm{Hz}<f<1 \mathrm{~Hz}$. Here $L_{\mathrm{L}}=2.5 \mathrm{Gm}$ is the LISA arm length, $f_{\mathrm{L}}=$ $c /\left(2 \pi L_{\mathrm{L}}\right)$ is the LISA arm transfer frequency, $S_{\mathrm{Lp}}=8.9 \times 10^{-23} \mathrm{~m}^{2} \mathrm{~Hz}^{-1}$ is the white position noise, and

$S_{\mathrm{a}}(f)=9 \times 10^{-30}\left[1+\left(10^{-4} \mathrm{~Hz} / f\right)^{2}+16\left(2 \times 10^{-5} \mathrm{~Hz} / f\right)^{10}\right] \mathrm{m}^{2} \mathrm{~s}^{-4} \mathrm{~Hz}^{-1}$,

is the colored acceleration noise level. This new LISA design sensitivity curve shows in both Fig. 1 and Fig. 2.

\subsection{Super-ASTROD}

Super-ASTROD (Super Astrodynamical Space Test of Relativity using Optical Devices) is a mission concept with four spacecraft in 5.2 AU orbits together with an Earth-Sun L1/L2 spacecraft ranging optically with one another to probe GWs with frequencies $0.1 \mu \mathrm{Hz}-1$ $\mathrm{mHz}$, to test fundamental laws of spacetime and to map the outer-solar-system mass distribution and dynamics [8]. In this paper, we address mainly the GW sensitivity of Super-ASTROD for enhancing the GW detection in the sensitivity gap $100 \mathrm{nHz}-10 \mu \mathrm{Hz}$. With three spacecraft in Jupiter-like solar orbits of radius $5.2 \mathrm{AU}$ and period of about 12 year, the desired baseline mission lifetime would be 25 years or more. The orbits of these 3 spacecraft are near the Sun-Jupiter L3, L4, L5 points respectively to form an ASTRODGW-like configuration [23, 24]. The Super-ASTROD spacecraft configuration will be inclined to the Jupiter orbit plane by $1-3^{\circ}$. The angular precession of the spacecraft configuration will be twice the Jupiter orbiting angular velocity around the Sun, i.e. about 6 years in period. In 6 years, the angular position of quasi-monochromatic GW sources will be resolved.

Due to its large extension, Super-ASTROD is a second-generation GW mission concept. To set its sensitivity goal, we review the GW sensitivity of ASTROD-GW $[23,24]$ in Fig. 1.

For ASTROD-GW, our goal on the instrumental strain psd amplitude is

$$
S_{\mathrm{An}}^{1 / 2}(f)=\left(1 / L_{\mathrm{A}}\right) \times\left\{\left[\left(1+0.5\left(f / f_{\mathrm{A}}\right)^{2}\right)\right] \times S_{\mathrm{Ap}}+\left[4 S_{\mathrm{a}} /(2 \pi f)^{4}\right]\right\}^{1 / 2} \mathrm{~Hz}^{-1 / 2},
$$


over the frequency range of $100 \mathrm{nHz}<f<1 \mathrm{~Hz}$. Here $L_{\mathrm{A}}=260 \times 10^{9} \mathrm{~m}$ is the ASTRODGW arm length, $f_{\mathrm{A}}=c /\left(2 \pi L_{\mathrm{A}}\right)$ is the ASTROD-GW arm transfer frequency, $S_{\mathrm{a}}=9 \times 10^{-30}$ $\mathrm{m}^{2} \mathrm{~s}^{-4} \mathrm{~Hz}^{-1}$ is the white acceleration noise level (the same as that for original LISA [21]), and $S_{\mathrm{Ap}}=10816 \times 10^{-22} \mathrm{~m}^{2} \mathrm{~Hz}^{-1}$ is the (white) position noise level due to laser shot noise which is $2704\left(=52^{2}\right)$ times that for original LISA [23]. The corresponding noise curve for the ASTROD-GW instrumental noise psd amplitude ${ }^{(\mathrm{MLDC})} S_{\mathrm{An}}{ }^{1 / 2}(f)$ with the same "reddening" factor as specified in MLDC program is

$$
{ }^{(\mathrm{MLDC})} S_{\mathrm{An}}{ }^{1 / 2}(f)=\left(1 / L_{\mathrm{A}}\right) \times\left\{\left[\left(1+0.5\left(f / f_{\mathrm{A}}\right)^{2}\right)\right] \times S_{\mathrm{Ap}}+\left[1+\left(10^{-4} / f\right)^{2}\right]\left(4 S_{\mathrm{a}} /(2 \pi f)^{4}\right)\right\}^{1 / 2} \mathrm{~Hz}^{-1 / 2},
$$

over the frequency range of $100 \mathrm{nHz}<f<1 \mathrm{~Hz}$. The one without reddening factor are shown with dashed line in the lower frequency part.

For Super-ASTROD, our goal on the instrumental strain noise psd amplitude is

$$
S_{\mathrm{Sn}}{ }^{1 / 2}(f)=\left(1 / L_{\mathrm{S}}\right) \times\left\{\left[\left(1+0.5\left(f / f_{\mathrm{S}}\right)^{2}\right)\right] \times S_{\mathrm{Sp}}+\left[4 S_{\mathrm{a}} /(2 \pi f)^{4}\right]\right\}^{1 / 2} \mathrm{~Hz}^{-1 / 2},
$$

over the frequency range of $100 \mathrm{nHz}<f<1 \mathrm{~Hz}$. Here $L_{\mathrm{S}}=1350 \times 10^{9} \mathrm{~m}(9 \mathrm{AU})$ is the nominal Super-ASTROD arm length, $f_{\mathrm{S}}=c /\left(2 \pi L_{\mathrm{S}}\right)$ is the Super-ASTROD arm transfer frequency, $S_{\mathrm{a}}=9 \times 10^{-30} \mathrm{~m}^{2} \mathrm{~s}^{-4} \mathrm{~Hz}^{-1}$ is the white acceleration noise level (the same as that for original LISA and ASTROD-GW), and $S_{\mathrm{Sp}}=291600 \times 10^{-22} \mathrm{~m}^{2} \mathrm{~Hz}^{-1}$ is the (white) position noise level due to laser shot noise which is $72900\left(=270^{2}\right)$ times that for original LISA.

\section{AMIGO}

A discussion of ground-based GW detector concepts to extend the present ground-based interferometers detection spectral range, i.e., the high-frequency GW band $10 \mathrm{~Hz}-100 \mathrm{~Hz}$ to middle-frequency band $0.1-10 \mathrm{~Hz}$ together with the plethora of potential astrophysical sources in this band is given in Harms et al. [27]. Harms et al. examine the potential sensitivity of three detection concepts (atom interferometers, torsion bar antennas and Michelson interferometers), estimate for their event rates and thereby, the sensitivity requirements for these detectors. They find that the scientific payoff from measuring astrophysical gravitational waves in this frequency band is great. However, although they find no fundamental limits to the detector sensitivity in this band, the remaining technical limits will be extremely challenging to overcome. In this conference, Ho Jung presents a middle-frequency GW detector concept SOGRO (Superconducting Omni-directional Gravitational Radiation Observatory) on Earth [28, 29]. The Newtonian-noise cancellation from infrasound and seismic surface fields is very challenging [30].

In this paper, we propose a first-generation middle-frequency mission concept AMIGO: Astrodynamical Middle-frequency Interferometric GW Observatory with the following specification:

Arm length: $10,000 \mathrm{~km}$ (or a few times of this)

Laser power: $2-10 \mathrm{~W}$

Acceleration noise: same as LPF has already achieved

Orbits and formation: 4 options (all LISA-like formations):

(i) Earth-like solar orbits (3-20 degrees behind the Earth orbit)

(ii) $600,000 \mathrm{~km}$ high orbit formation around the Earth

(iii) $100,000 \mathrm{~km}-250,000$ high orbit formation around the Earth

(iv) near Earth-Moon L4 (or L5) halo orbit formation 
The Scientific Goals of AMIGO are: to bridge the spectra gap between first-generation high-frequency and low-frequency GW sensitivities; Detecting intermediate mass $\mathrm{BH}$ coalescence; Detecting inspiral phase and predict time of binary black hole coalescence together with neutron star coalescence for ground interferometers; Detecting compact binary inspirals for studying stellar evolution and galactic population.

For AMIGO, our baseline on the noise psd amplitude assuming $2 \mathrm{~W}$ laser power, $30 \mathrm{~cm}$ telescopes and same acceleration noise as new LISA is:

$S_{\mathrm{AMIGOn}}{ }^{1 / 2}(f)=(20 / 3)^{1 / 2}\left(1 / L_{\mathrm{AMIGO}}\right) \times\left[\left(1+\left(f /\left(1.29 f_{\mathrm{AMIGO}}\right)\right)^{2}\right)\right]^{1 / 2} \times\left[\left(S_{\mathrm{AMIGOp}}+4 S_{\mathrm{a}} /(2 \pi f)^{4}\right)\right]^{1 / 2} \mathrm{~Hz}^{-1 / 2},(6)$

over the frequency range of $20 \mu \mathrm{Hz}<f<1 \mathrm{kHz}$. Here $L_{\mathrm{AMIGO}}=0.01 \times 10^{9} \mathrm{~m}$ is the AMIGO arm length, $f_{\mathrm{AMIGO}}=c /\left(2 \pi L_{\mathrm{AMIGO}}\right)$ is the AMIGO arm transfer frequency, $S_{\mathrm{AMIGOp}}$ $=1.424 \times 10^{-28} \mathrm{~m}^{2} \mathrm{~Hz}^{-1}$ is the (white) position noise level due to laser shot noise which is $16 \times 10^{-6}\left(=0.004^{2}\right)$ times that for new LISA. $S_{\mathrm{a}}(f)$ is the same colored acceleration noise level in (2). The AMIGO baseline sensitivity (6) is plotted as AMIGO dashed curve in both Fig. 1 and Fig. 2.

Since power and lower shot noise is crucial in reach better sensitivity in middle part of the sensitivity curve, we use $10 \mathrm{~W}$ laser power and $36 \mathrm{~cm} \phi$ as our design values of the AMIGO mission concept to gain a factor of $10\left[\approx(10 / 2) \times(36 / 30)^{4}\right]$ for shot noise design sensitivity. The AMIGO design sensitivity (6) is plotted as AMIGO solid curve in both Fig. 1 and Fig. 2 by using $S_{\text {AMIGOp }}=0.1424 \times 10^{-28} \mathrm{~m}^{2} \mathrm{~Hz}^{-1}$.

In Fig. 1 and Fig. 2, the strain psd amplitude of GW150914 is calculated from its characteristic amplitude in Figure 1 of [25] using standard formula of conversion. AMIGO with either baseline sensitivity or design sensitivity would detect the inspiral phase of GW150914 and predict the coalescence time for the benefit of doing multi-messenger astronomy. However, the design sensitivity has better coverage in detecting the inspiral phase of neutron star coalescence events.

The numerical TDIs for AMIGO would be easier to design compared to new LISA due to AMIGO's shorter arm length. X, Y, Z TDI configurations are well suited for AMIGO. However, experimental requirement on TDI is more stringent and needs developments. More studies on the mission concept and various choices of orbit design are under preparation [31].

\section{Discussion and outlook}

LIGO's measurement of GWs from black hole coalescence fully ushered us into the era of GW astronomy. LISA space GW mission will explore the GW sources of large part of our Universe with large $\mathrm{S} / \mathrm{N}$ ratio in the low-frequency part. On the even lower frequency side, PTAs are actively looking for GWs. Between the frequency bands sensitive to PTAs and to first generation space GW detector, Super-ASTROD is discussed as a secondgeneration space detector concept to have better sensitivity in the interim frequency band to explore GW sources.

Currently, a number of detection methods are proposed and under active research to bridge the middle-frequency band gap between Earth-based and space-borne GW observations with important science goals. In this band, technical limits will be extremely challenging to overcome for Earth-based due to Newtonian noises. In this paper, we propose a first-generation middle-frequency mission concept AMIGO with 10,000 km arm length. The technical readiness level is high. The sensitivity is good to reach science goals considered in the last section.

If a pathfinder mission is desired with 2 -spacecraft demonstration of ranging in the solar-system for a LISA-like mission, the case with 2-5 degrees lagging behind the Earth of 
the first orbit choice in the last section could be considered. Just take one arm of this AMIGO case, it would be good to test many things in the solar system: deployment, both radio and laser communications, noise budget, and drag-free system together with a concentrated effort on distance metrology. It might be simpler than go to L1 or L2 SunEarth Lagrange point.

I would like to thank Ling-Wei Luo for his help in drawing Figure 1 and Figure 2.

\section{References}

1. B. P. Abbott, et al. (LIGO Scientific and Virgo Collaborations), Observation of gravitational waves from a binary black hole merger, Phys. Rev. Lett. 116, 061102 (2016)

2. B. P. Abbott et al. (LIGO Scientific and Virgo Collaborations), GW151226: Observation of gravitational waves from a 22 -solar-mass binary black hole coalescence, Phys. Rev. Lett. 116, 241103 (2016)

3. B. P. Abbott et al. (LIGO Scientific and Virgo Collaborations), GW170104: Observation of a 50-solar-mass binary black hole coalescence at redshift 0.2 Phys. Rev. Lett. 118, 221101 (2017)

4. K. Kuroda, W.-T. Ni and W.-P. Pan, Gravitational waves: Classification, methods of detection, sensitivities, and sources, Int. J. Mod. Phys. D 24, 1530031 (2015); also in One Hundred Years of General Relativity: From Genesis and Empirical Foundations to Gravitational Waves, Cosmology and Quantum Gravity, Chap. 10, ed. W.-T. Ni (World Scientific, Singapore, 2017)

5. W.-T. Ni, Gravitational wave, dark energy and inflation, Mod. Phys. Lett. A 25, 922 (2010); arXiv:1003.3899; Classification of Gravitational Waves, http://astrod.wikispaces.com/file/view/GW-classification.pdf

6. D. Blair, et al., Gravitational wave astronomy: the current status, Sci. China-Phys. Mech. Astron. 58, 120402 (2015)

7. S. Kuroyanagi, L.-W. Luo and W.-T. Ni, Gravitational wave sensitivities over all frequency bands, paper in preparation

8. W.-T. Ni, Super-ASTROD: Probing primordial gravitational waves and mapping the outer solar system, Class. Quantum Grav. 26, 075021 (2009)

9. M. Armano, H. Audley, G. Auger et al., Sub-Femto-g Free Fall for Space-Based Gravitational Wave Observatories: LISA Pathfinder Results, Phys. Rev. Lett. 116, 231101 (2016)

10. A. Petiteau, M. Hewitson, G. Heinzel, E. Fitzsimons and H. Halloin, LISA noise budget, Tech. rep. LISA Consortium IISA-CST-TN-0001 (2016)

11. P. Amaro-Seoane, H. Audley, S. Babak et al., Laser Interferometer Space Antenna, submitted to ESA on January 13th in response to the call for missions for the L3 slot in the Cosmic Vision Programme, arXiv:1702.00786 [astro-ph.IM]

12. M. Tinto and S. V. Dhurandhar, Time-delay interferometry, Liv. Rev. Rel. 17, 6 (2014); and references therein

13. G. Wang and W.-T. Ni, Numerical simulation of time delay interferometry for new LISA, TAIJI and other LISA-like missions, arXiv:1707.09127 [astro-ph.IM]

14. G. de Vine, B. Ware, K. McKenzie, R. E. Spero, W. M. Klipstein, and D. A. Shaddock, Experimental Demonstration of Time-Delay Interferometry for the Laser Interferometer Space Antenna, Phys. Rev. Lett. 104, 211103 (2010) 
15. S. J. Mitryk, G. Mueller and J. Sanjuan, Hardware-based demonstration of time-delay interferometry and TDI-ranging with spacecraft motion effects, Phys. Rev. D 86, 122006 (2012)

16. A.-C. Liao, W.-T. Ni and J.-T. Shy, On the study of weak-light phase locking for laser astrodynamical missions (in Chinese) Publications of the Yunnan Observatory 3, 88100 (2002)

17. A.-C. Liao, W.-T. Ni and J.-T. Shy, Pico-watt and femto-watt weak-light phase locking Int. J. Mod. Phys. D 11, 1075-1085 (2002)

18. G. J. Dick, M. D. Strekalov, K. Birnbaum et al., Optimal phase lock at femtowatt power levels for coherent optical deep-space transponder, IPN Progress Report 42 1752008 (2008)

19. O. Gerberding, B. Sheard, I. Bykov et al., Phasemeter core for intersatellite laser heterodyne interferometry: modelling, simulations and experiments, Classical Quantum Gravity 30, 235029 (2013)

20. S. P. Francis, T. T.-Y. Lam, K. McKenzie et al., Weak-light phase tracking with a low cycle slip rate, Optics Letters 39, 5251-5154 (2014)

21. LISA Study Team, LISA (Laser Interferometer Space Antenna) - A Cornerstone Mission for the Observation of Gravitational Waves, ESA System and Technology Study Report, ESA-SCI 11 (2000)

22. W.-T. Ni, ASTROD and gravitational waves, pp. 117-129 in Gravitational Wave Detection, edited by K. Tsubono, M.-K. Fujimoto and K. Kuroda (Universal Academy Press, Tokyo, Japan, 1997)

23. W.-T. Ni, ASTROD-GW: Overview and progress, Int. J. Mod. Phys. D 22, 1431004 (2013); and references therein

24. W.-T. Ni, Gravitational Wave Detection in Space, Int. J. Mod. Phys. D 25, 1630001 (2016); also in One Hundred Years of General Relativity: From Genesis and Empirical Foundations to Gravitational Waves, Cosmology and Quantum Gravity, Chap. 12, ed. W.-T. Ni (World Scientific, Singapore, 2017); and references therein

25. A. Sesana, Prospects for multiband gravitational-wave astronomy after GW150914, Phys. Rev. Lett. 116, 231102 (2016)

26. N. Cornish and T. Robson, Galactic binary science with the new LISA design, J. Phys.: Conf. Ser. 840, 012024 (2017)

27. J. Harms, B. J. J. Slagmolen, R. X. Adhikari et al, Low-frequency terrestrial gravitational-wave detectors, Phys. Rev. D 88, 122003 (2013)

28. H. J. Paik, SOGRO (Superconducting Omni-directional Gravitational Radiation Observatory), Plenary talk given in Joint Meeting of 13th International Conference on Gravitation, Astrophysics, and Cosmology and 15th Italian-Korean Symposium on Relativistic Astrophysics, Ewha Womans University, Seoul, Korea, July 3-7, 2017

29. H. J. Paik, C. E Griggs, M. Moody et al., Low-frequency terrestrial tensor gravitational-wave detector, Class. Quantum Grav. 33, 075003 (19pp) (2016)

30. J. Harms and H. J. Paik, Newtonian-noise cancellation in full-tensor gravitational-wave detectors, Phys. Rev. D 92, 022001 (2015)

31. W.-T. Ni, G. Wang and A.-M. Wu, Astrodynamical Middle-frequency Interferometric GW Observatory AMIGO: mission concept and orbit design, paper in preparation 